\title{
Crecimiento del caimán del Orinoco (Crocodylus intermedius, Crocodylia: Crocodylidae) en dos condiciones de cautiverio
}

\author{
Aldeima T. Pérez T. \\ Instituto Limnológico, Universidad de Oriente (U.D.O), Caicara del Orinoco, estado Bolívar, Venezuela; \\ aldeimaperez@hotmail.com
}

\author{
Recibido 24-IV-2006. Corregido 27-II-2007. Aceptado 25-VII-2007.
}

\begin{abstract}
Growth of the Orinoco Caiman (Crocodylus intermedius, Crocodylia: Crocodylidae) under two captivity conditions. In order to determine the growth of Caiman of the Orinoco (Crocodylus intermedius) under two conditions of captivity, 40 specimens were raised during 11 months and 15 days in two circular tanks, with $28.3 \mathrm{~m}^{2}$ of surface area and a volume of $62.2 \mathrm{~m}^{3}$ in each tank. The tanks were built with concrete walls and guarded blocks covered internally with sheets of myrrhlike resin, and a roof of galvanized sheets. One tank was covered partially with the galvanized sheets (tank I), the other was totally covered (tank II). Twenty caimans were placed in each tank, and both groups were fed with $85 \%$ beef, $10 \%$ fresh fish, $5 \%$ hen eggs and a mixture of minerals and vitamins. The length and weight differed significantly between the groups $(p<0.001)$. Mean growth $(103.0 \pm 6.81 \mathrm{~cm})$ and weight $(3987 \pm 0.98 \mathrm{~g})$ were higher in tank II, (tank I: $88.9 \pm 7.58 \mathrm{~cm} ; 2705 \pm$ $0.69 \mathrm{~g})$. The greater growth in tank II reflects higher air and water temperatures. The survival rate was $97.5 \%$. These results can be used for rearing caimans in captivity for conservation and commercial purposes. Rev. Biol. Trop. 56 (1): 349-354. Epub 2008 March 31.
\end{abstract}

Key words: captivity, Crocodylus intermedius, growth, Venezuela.

El órden Crocodylia está dividido en tres grupos básicos considerados como subfamilias. La Alligatorinae incluye cuatro géneros con especies sobrevivientes: Alligator, el cual contiene dos especies; Caiman, definido con dos especies; Paleosuchus, con dos especies de caimanes enanos y Melanosuchus, el caimán negro. La subfamilia Crocodylinae está conformada por tres géneros con especies sobrevivientes: Crocodylus, los verdaderos cocodrilos con 12 especies; Osteolaemus, el cocodrilo enano y Tomistoma, el falso gavial y Gavialinae que contiene sólo un género con una especie sobreviviente, el gharial, Gavialis gangeticus (Ross y Magnusson 1989).

En la actualidad, el órden está compuesto por 22 especies de las cuales cinco se encuentran en Venezuela: Caiman crocodilus (baba), Paleosuchus palpebrosus y
Paleosuchus trigonatus (caimanes enanos), Crocodylus intermedius (caimán del Orinoco) y Crocodylus acutus (caimán de la costa). El caimán del Orinoco está restringido a la cuenca del río Orinoco en Venezuela y a la región este de Colombia (Brazaitis 1973). Las poblaciones naturales de $C$. intermedius, disminuyeron debido a la caza comercial entre los años 1930 y 1950 (Medem 1981). La cacería excesiva y la destrucción del hábitat natural parecen ser los factores que más han incidido en la reducción de las poblaciones de $C$. intermedius (Brazaitis 1973). Actualmente la especie está catalogada en peligro de extinción e incluida en el Apéndice I de la Ley Aprobatoria de la Convención internacional sobre el Comercio de Especies Amenazadas de Fauna y Flora Silvestres (CITES 1985). La cría en cautiverio y posterior liberación de los crocodílidos ha 
sido llevada a cabo en varias partes del mundo, como una de las estrategias destinadas a la recuperación de sus poblaciones (Pooley 1991), sin embargo, la transición de estos reptiles de su hábitat nativo, a un ambiente artificial representa un gran desafío en términos de manejo y nutrición (McNease y Joanen 1981).

Se han realizado estudios sobre la dieta y el crecimiento del caimán del Orinoco en cautiverio (Pérez y Velasco 2002), sin embargo, la información que se tiene sobre las condiciones óptimas para la cría de esta especie es limitada.

El objetivo del presente trabajo fue evaluar el crecimiento del caimán del Orinoco (Crocodylus intermedius) en dos condiciones de cautiverio como alternativa a la repoblación de los cuerpos de aguas naturales.

\section{MATERIALES Y MÉTODOS}

La crianza de Crocodylus intermedius se llevó a cabo en dos tanques circulares; cada uno con una superficie de $28.3 \mathrm{~m}^{2}, 2.20 \mathrm{~m}$ de altura, un volumen de $62.2 \mathrm{~m}^{3}$, una pendiente del $2 \%$ hacia el desagüe central y una profundidad del agua de $21 \mathrm{~cm}$. Los tanques estaban construidos de bloque y piso de concreto pulido, y presentaban un techo de láminas galvanizadas e internamente cubiertos con láminas de resina ("anime"). El tanque I fue cubierto parcialmente con láminas galvanizadas permitiendo la penetración de luz solar, mientras que el tanque II se techó totalmente para protegerlo de los rayos del sol. Cada tanque poseía una puerta de entrada y previo a ésta un pediluvio (lava pié) y un punto de agua blanca para el lavado interno (Pérez 2000). Los tanques se lavaron diariamente con una solución desinfectante a base de Yodo (IOSAN o IOSEP) empleando un cepillo para cada uno. Cada tanque fue enjuagado y llenado nuevamente con agua limpia. El uso de baños desinfectantes en los pediluvios fue necesario, para evitar el transporte de bacterias u otros organismos patógenos a los tanques.

Los jóvenes de caimán del Orinoco se obtuvieron del Fundo Pecuario Masaguaral, estado Guárico, Venezuela. Se colocaron 20 cocodrilos en el tanque I con una longitud y peso promedio de $39.1 \pm 1.38 \mathrm{~cm}$ y $208.3 \pm$ 22.1 g y 20 individuos con un promedio de $38.4 \pm 1.45 \mathrm{~cm}$ y $180.3 \pm 24.8 \mathrm{~g}$ en el tanque II. Cada 30 días, y por un período de nueve meses y 15 días, se les midió la longitud total o cabeza-cola (LT) y el peso, utilizando una cinta métrica de $150 \mathrm{~cm}$ y $1 \mathrm{~mm}$ de precisión y una balanza de $0.1 \mathrm{~g}$ de precisión y $6100 \mathrm{~g}$ de capacidad, respectivamente. Durante abril y mayo el crecimiento no fue evaluado debido al estado de agresión que presentaron los animales.

La alimentación consistió en una dieta conformada por un $85 \%$ de carne de res, $10 \%$ de pescado, $5 \%$ de huevos de gallinas complementadas con mezcla de minerales (calcio, fósforo, sodio, zinc, cobalto, azufre, selenio, yodo, flúor, magnesio, cobre y manganeso en una proporción de $4 \mathrm{~g}$ por cada $100 \mathrm{~g}$ de alimento) y vitaminas $\left(\mathrm{A}, \mathrm{B}_{1}, \mathrm{~B}_{2}, \mathrm{~B}_{6}, \mathrm{~B}_{12}, \mathrm{C}\right.$, $\mathrm{D}_{3}, \mathrm{E}, \mathrm{K}$, ácido fólico, ácido nicotínico en una proporción de $1 \mathrm{~g}$ por cada $100 \mathrm{~g}$ de alimento). Las proteínas de la dieta fueron molidas y colocadas en los tanques en pequeños montículos para que los cocodrilos comieran $a d$ libitum. La alimentación fue suministrada diariamente, luego de pesar la cantidad del alimento ofrecido, en horas de la tarde (14 h). La temperatura del agua y del aire dentro de los tanques fue registrada una vez al día y a primeras horas de la tarde (13:30 h), utilizando un termómetro ambiental de $36 \mathrm{~cm}$ de largo, un ámbito de medición de $-10^{\circ} \mathrm{C}$ a $200^{\circ} \mathrm{C}$ $\left( \pm 1^{\circ} \mathrm{C}\right)$. Para medir la temperatura del aire el termómetro se colocó dentro de los tanques a $90 \mathrm{~cm}$ del techo de lámina galvanizada. $\mathrm{La}$ temperatura del agua se midió a $10 \mathrm{~cm}$ de profundidad entre el fondo y la superficie del agua en cada tanque.

Análisis estadístico: Los datos del crecimiento en longitud y peso fueron evaluados y comparados entre los diferentes tipos de tanques mediante la prueba $\mathrm{T}$ de Student. Las variaciones de la temperatura mensual del aire y del agua, y entre los tanques de cría, se determinó a través de un análisis de varianza de dos vías (Modelo I), con ayuda del software SGPLUS 
y la comparación de los promedios según la prueba a posteriori Duncan $(p<0.05)$.

\section{RESULTADOS}

Después de 11 meses y 15 días de cría, los ejemplares del tanque I alcanzaron una longitud total promedio de $88.9 \pm 7.58 \mathrm{~cm}$, mientras que los individuos del tanque II midieron $103.0 \pm 6.81 \mathrm{~cm}$ (Cuadro 1). En el tanque I el $47.3 \%$ de los ejemplares midieron más de 80 $\mathrm{cm}$ de longitud total. El individuo de mayor talla alcanzó $103.0 \mathrm{~cm}$ y el de mayor peso 4 $000 \mathrm{~g}$. En el tanque II el $75 \%$ de los individuos alcanzaron más de $100 \mathrm{~cm}$ de longitud total. El organismo de mayor tamaño alcanzó $113.8 \mathrm{~cm}$ y el de mayor peso $5750 \mathrm{~g}$. El peso promedio final de los cocodrilos fue $2705 \pm 0.69 \mathrm{~g}$ y $3987 \pm 0.98 \mathrm{~g}$ respectivamente (Cuadro 2).
Los individuos del tanque II incrementaron su longitud total y peso en una tasa mayor que la que alcanzaron los cocodrilos del tanque I. Se encontró diferencias significativas en el crecimiento en longitud total (prueba $\mathrm{t}=-6.1, p<$ 0.001 ) y peso (prueba $\mathrm{t}=-4.7, p<0.001$ ) de los ejemplares en relación a las dos condiciones de cría. En el tanque I, la tasa de crecimiento del caimán del Orinoco osciló de 16.6 a 29.6 $\mathrm{mm} /$ día y 15.88 a $90.2 \mathrm{~g} /$ día, mientras que en el tanque II el crecimiento varió entre 17.6 a 34.3 $\mathrm{mm} /$ día y 17.7 a $132.9 \mathrm{~g} /$ día.

La temperatura del aire y del agua entre los tanques I y II varió significativamente $(p$ $<0.05$ ). En el tanque I se registraron las máximas temperaturas promedios del aire durante febrero (36.3, S.E. = 0.33), marzo $(36.5$, S.E. $=$ $0.36)$ y abril $(37.2$, S.E. $=0.40)$ mientras que en agosto (33.7, S.E. $=0.55)$, noviembre (34.3, S.E. $=0.20)$ y diciembre $(33.8$, S.E. $=0.24)$

CUADRO 1

Promedio final de crecimiento en longitud total ( $\mathrm{cm}$ ) de C. intermedius en dos condiciones de cautiverio

\begin{tabular}{|c|c|c|c|c|c|}
\hline Tanque & $\mathrm{N}^{1}$ & Ámbito & Promedio de longitud total final $(\mathrm{cm})$ & $\mathrm{Sx}^{2}$ & $t^{3}$ \\
\hline \multirow[t]{2}{*}{$\mathrm{I}$} & 19 & $77.5-103$ & 88.9 & 7.58 & \\
\hline & & & & & $-6.0916^{* * * 4}$ \\
\hline II & 20 & $90.5-113.8$ & 103.0 & 6.81 & \\
\hline
\end{tabular}

CUADRO 2

Promedio final de crecimiento en peso $(g)$ de C. intermedius en dos condiciones de cautiverio

\begin{tabular}{|c|c|c|c|c|c|}
\hline Tanque & $\mathrm{N}^{1}$ & Ámbito & Promedio de peso final (g) & $\mathrm{Sx}^{2}$ & $t^{3}$ \\
\hline \multirow[t]{2}{*}{ I } & 19 & $1800-4000$ & 2705 & 0.69 & \\
\hline & & & & & $-4.71263^{* * *}$ \\
\hline II & 20 & $2300-5750$ & 3987 & 0.98 & \\
\hline $\begin{array}{l}\mathrm{N}= \\
\mathrm{S}_{\mathrm{x}}= \\
\mathrm{t}=\mathrm{pr} \\
* * *\end{array}$ & de & nes. & 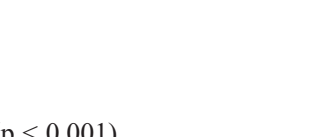 & & \\
\hline
\end{tabular}


se obtuvieron los menores valores $(p<0.05)$ (Cuadro 3). En el tanque II, las mayores temperaturas promedios del aire correspondieron a septiembre $(40.8$, S.E. $=0.42)$, octubre $(40.3$, S.E. $=0.40) \mathrm{y}$ abril $(41.0$, S.E. $=0.49)$, en contraste con las encontradas en agosto (37.9, S.E. $=0.64)$, diciembre $(38.1$, S.E. $=0.24)$ y enero $(39.0, \mathrm{~S} . \mathrm{E}=0.24)$ donde se registró los valores más bajos.

En el tanque I, las mayores temperaturas promedio del agua se registraron en agosto $(33.6$, S.E. $=0.38)$ y septiembre $(33.4$, S.E. $=0.30)$ diferenciándose significativamente de los valores promedio alcanzados en diciembre $(28.7$, S.E. $=0.13)$ y enero $(28.6$, S.E. $=$ 0.13) $(p<0.05)$. En el tanque II los valores máximos se observaron en septiembre (34.6, S.E. $=0.15)$, octubre $(34.9$, S.E. $=0.17)$ y abril $(34.4$, S.E. $=0.24)$, en comparación con los promedios menores de agosto (32.6, S.E. = $0.23)$ y diciembre $(32.9$, S.E. $=0.09)(p<0.05)$ (Cuadro 4).

Los neonatos y juveniles de Crocodylus intermedius fueron muy sensibles al stress provocado por ruidos, excesiva actividad en los tanques de cría y especialmente por el manejo requerido para capturarlos y medirlos. El manejo de los cocodrilos produjo sofocación, combates y daños físicos, causando lesiones en la cola, boca, ojos y superficie del cuerpo. La tasa de supervivencia durante el estudio fue $97.5 \%$.

\section{DISCUSIÓN}

Medem (1981) registró para el caimán del Orinoco un crecimiento promedio de $0.6 \pm 0.24$ $\mathrm{mm} /$ día y $4.04 \mathrm{~g}$ /día; mientras que RamírezPerilla (1991) evaluaron un crecimiento promedio diario de $1.24 \mathrm{~mm}$ y $27.7 \mathrm{~g}$ de peso para esta especie. Ramo et al. (1992) reportaron para Crocodylus intermedius en cautiverio un crecimiento de $39 \mathrm{~mm} /$ día en machos y 33 $\mathrm{mm} /$ día en hembras. Blohm (1973) señaló que los cocodrilos y caimanes pueden alcanzar $0.89 \mathrm{~mm} /$ día. Posiblemente la variabilidad en los valores de crecimiento en longitud total y peso obtenidos en el presente estudio, pueden estar relacionados con la temperatura del agua y del aire dentro del tanque de cría (diseño del

CUADRO 3

Promedio de Temperatura del aire $\left({ }^{\circ} \mathrm{C}\right)$ en dos condiciones de cautiverio

\begin{tabular}{|c|c|c|c|c|c|c|c|c|}
\hline & & $\begin{array}{l}\text { Tanque } \\
\text { (Con luz }\end{array}$ & $\begin{array}{c}\text { I } \\
\text { solar) }\end{array}$ & & & $\begin{array}{l}\text { Tanque } \\
\text { (Sin luz }\end{array}$ & $\begin{array}{c}\text { II } \\
\text { Solar) }\end{array}$ & \\
\hline Mes & $\mathrm{N}^{1}$ & Ámbito & $X^{2}$ & $\mathrm{~S}_{\mathrm{X}}^{3}$ & $\mathrm{~N}^{1}$ & Ámbito & $X^{2}$ & $\mathrm{~S}_{\mathrm{X}}^{3}$ \\
\hline Agosto & 25 & $27.0-37.0$ & $33.7^{\mathrm{E}}$ & 0.55 & 25 & $29.5-41.5$ & $37.9^{\mathrm{D}}$ & 0.64 \\
\hline Septiembre & 23 & $30.5-38.0$ & $36.0^{\mathrm{B}}$ & 0.35 & 23 & $34.0-43.0$ & $40.8^{\mathrm{A}}$ & 0.42 \\
\hline Octubre & 25 & $31.9-38.0$ & $35.5^{\mathrm{BC}}$ & 0.31 & 25 & $35.0-43.5$ & $40.3^{\mathrm{AB}}$ & 0.40 \\
\hline Noviembre & 25 & $31.8-36.0$ & $34.3 \mathrm{DE}$ & 0.20 & 25 & $35.5-41.0$ & $39.2 \mathrm{BC}$ & 0.24 \\
\hline Diciembre & 23 & $31.5-36.1$ & $33.8^{\mathrm{E}}$ & 0.24 & 23 & $34.5-39.5$ & $38.1^{\mathrm{CD}}$ & 0.24 \\
\hline Enero & 21 & $33.0-38.8$ & $35.0^{\mathrm{CD}}$ & 0.28 & 21 & $36.8-42.8$ & $39.0^{\mathrm{BCD}}$ & 0.24 \\
\hline Febrero & 24 & $32.7-39.0$ & $36.3^{\mathrm{AB}}$ & 0.33 & 24 & $36.0-43.2$ & $40.2 \mathrm{AB}$ & 0.38 \\
\hline Marzo & 26 & $30.0-39.8$ & $36.5^{\mathrm{AB}}$ & 0.36 & 26 & $33.0-42.7$ & $40.2^{\mathrm{AB}}$ & 0.36 \\
\hline Abril & 19 & $33.0-40.0$ & $37.2^{\mathrm{A}}$ & 0.40 & 19 & $36.8-45.0$ & $41.0^{\mathrm{A}}$ & 0.49 \\
\hline
\end{tabular}

$1 \quad \mathrm{~N}=$ Número de observaciones.

$2 \mathrm{X}=$ promedio. Duncan $(\mathrm{p}<0.05)$.

$3 \quad \mathrm{~S}_{\mathrm{x}}=$ error estándar. 
CUADRO 4

Promedio de Temperatura del agua $\left({ }^{\circ} \mathrm{C}\right)$ en dos condiciones de cautiverio

$\begin{array}{lcccccccc} & & \begin{array}{c}\text { Tanque } \\ \text { (Con luz }\end{array} & \begin{array}{c}\mathrm{I} \\ \text { solar) }\end{array} & & & \begin{array}{c}\text { Tanque } \\ \text { (Sin luz }\end{array} & \begin{array}{c}\text { II } \\ \text { Solar) }\end{array} \\ \text { Mes } & \mathrm{N}^{1} & \text { Ámbito } & \mathrm{X}^{2} & \mathrm{~S}_{\mathrm{X}}{ }^{3} & \mathrm{~N}^{1} & \text { Ámbito } & \mathrm{X}^{2} & \mathrm{~S}_{\mathrm{X}}{ }^{3} \\ \text { Agosto } & 25 & 29.0-36.0 & 33.6^{\mathrm{A}} & 0.38 & 25 & 30.0-34.0 & 32.6^{\mathrm{F}} & 0.23 \\ \text { Septiembre } & 23 & 30.0-36.0 & 33.4^{\mathrm{A}} & 0.30 & 23 & 32.5-35.5 & 34.6^{\mathrm{A}} & 0.15 \\ \text { Octubre } & 25 & 29.8-33.5 & 31.8^{\mathrm{C}} & 0.20 & 25 & 32.5-36.0 & 34.9^{\mathrm{A}} & 0.17 \\ \text { Noviembre } & 25 & 29.0-31.5 & 30.0^{\mathrm{D}} & 0.11 & 25 & 31.5-35.0 & 33.9^{\mathrm{BC}} & 0.13 \\ \text { Diciembre } & 23 & 27.5-30.0 & 28.7^{\mathrm{E}} & 0.13 & 23 & 32.0-33.5 & 32.9^{\mathrm{EF}} & 0.09 \\ \text { Enero } & 21 & 27.5-30.0 & 28.6^{\mathrm{E}} & 0.13 & 21 & 32.0-34.8 & 33.3^{\mathrm{DE}} & 0.12 \\ \text { Febrero } & 24 & 28.2-31.0 & 29.9^{\mathrm{D}} & 0.15 & 24 & 32.2-35.5 & 34.0^{\mathrm{BC}} & 0.17 \\ \text { Marzo } & 26 & 29.0-32.5 & 30.5^{\mathrm{D}} & 0.17 & 26 & 31.0-35.0 & 33.7^{\mathrm{CD}} & 0.15 \\ \text { Abril } & 19 & 31.0-35.2 & 32.7^{\mathrm{B}} & 0.24 & 19 & 31.8-36.0 & 34.4^{\mathrm{AB}} & 0.24\end{array}$

$1 \quad \mathrm{~N}=$ Número de observaciones.

$2 \mathrm{X}=$ promedio. Duncan $(\mathrm{p}<0.05)$.

$3 \quad \mathrm{~S}_{\mathrm{x}}=$ error estándar.

tanque), la calidad del alimento ofrecido, condiciones geográficas, entre otros factores que pudieran determinar el crecimiento de estos organismos en cautiverio.

Se han realizado estudios para demostrar la importancia de mantener a los crocodílidos recién nacidos y juveniles a temperaturas altas, en ámbitos de 20 a $32^{\circ} \mathrm{C}$, para acelerar las funciones metabólicas, estimulando así al consumo de alimento (Coulson et al. 1973, Joanen y McNease 1979, Staton et al. 1986). Medem (1981) determinó que la tasa de crecimiento del caimán del Orinoco depende principalmente de la temperatura del agua y del ambiente y la eficiencia biológica depende mayormente de la calidad del alimento y al incremento de la tasa metabólica de los cocodrilos que se logra con mayores temperaturas ambientales. Asimismo, Pooley (1991) señaló que a temperaturas bajas, el consumo de alimento desciende drásticamente ya que la acción de ciertas enzimas digestivas y el metabolismo corporal disminuyen. Pinheiro et al. (1992) estudiaron el efecto de la temperatura del agua en el crecimiento inicial de 20 ejemplares de Caiman crocodilus yacare, y determinaron que a temperaturas del agua entre $29.5^{\circ} \mathrm{C}$ y $32^{\circ} \mathrm{C}$, la tasa de crecimiento fue mayor.

En conclusión, en condiciones óptimas de cautiverio Crocodylus intermedius alcanza un crecimiento diario entre 17.6 a $34.3 \mathrm{~mm}$ y 17.7 a 132.9 g durante los primeros 11 meses de vida. En el tanque totalmente techado y protegido de la luz solar se obtuvo el mayor crecimiento de $C$. intermedius debido al mantenimiento de los niveles de temperatura del aire y del agua superior a los correspondientes al tanque parcialmente techado y con penetración de luz solar.

\section{AGRADECIMIENTOS}

A la Fundación para el Desarrollo de la Ciencia y la Tecnología de la Región Guayana (FUNDACITE-Guayana) por el apoyo económico para este estudio. Al Ministerio del Ambiente y de los Recursos Naturales, Dirección General de Fauna (PROFAUNA) por su constante y valiosa colaboración para 
la obtención y transporte de los cocodrilos. A Thomas Blohm del Hato Masaguaral por el aporte de los cocodrilos.

\section{RESUMEN}

para determinar el crecimiento del caimán del Orinoco (Crocodylus intermedius), en diferentes condiciones de cautiverio, se criaron 40 ejemplares durante 11 meses y 15 días. Se utilizaron dos tanques circulares, con una superficie de $28.3 \mathrm{~m}^{2}$ y un volumen de $62.2 \mathrm{~m}^{3}$, construidos con bloques de concreto y protegidos con techo de láminas galvanizadas e internamente cubiertos con láminas de resina. Un tanque fue cubierto parcialmente con láminas galvanizadas (Tanque I) y el otro se techó totalmente (Tanque II). Se colocaron 20 cocodrilos en cada tanque y a los dos grupos se les administró una dieta compuesta por $85 \%$ de carne de res, $10 \%$ de carne de pescado, $5 \%$ de huevos de gallina y mezcla de minerales y vitaminas. El crecimiento en longitud total y peso difirió entre grupos ( $p$ $<0.001$ ). Los cocodrilos del tanque II obtuvieron un mayor crecimiento promedio en longitud total $(103.0 \pm 6.81 \mathrm{~cm})$ y peso (3 $987 \pm 0.98 \mathrm{~g})$. En el tanque los valores fueron $88.9 \pm 7.58 \mathrm{~cm}$; y $2705 \pm 0.69 \mathrm{~g}$. El mayor crecimiento en el tanque II se debió a mayores niveles de temperatura del aire y del agua. La tasa de supervivencia fue $97.5 \%$. La investigación demostró la factibilidad de criar cocodrilos en estas condiciones de cautiverio para la conservación y para propósitos comerciales.

Palabras clave: cautiverio, crecimiento, Crocodylus intermedius, Venezuela.

\section{REFERENCIAS}

Blohm, T. 1973. Conveniencia de criar crocodílidos en Venezuela con fines económicos y para prevenir su extinción. Simposio Internacional sobre Fauna Silvestre y Pesca Fluvial y Lacustre Amazónica. Manaus, Brazil.

Brazaitis, P. 1973. The identification of living crocodilians. Zoologica 58: 59-101.

CITES, 1985. Manual de identificación. Reptilia, Amphibia, Pisces, p. 60. In P. Dollinger (ed.). Secretaría de la Convención Internacional de Tráfico de Especies Amenazadas de Flora y Fauna Silvestres. PNUMA, Gland, Suiza.
Coulson, T.D., R.A. Coulson \& T. Hernandez. 1973. Some observations on the growth of captive alligators. Zoologica 58: 47-52.

Joanen, T. \& L McNease. 1979. Culture of the american Alligator. Intl. Zoo. Yearbook 19: 61-66.

McNease, L. \& T. Joanen. 1981. Nutrition of alligators. Proc. Alligator Production Conf. Gainesville, Florida. 1: $15-28$.

Medem, F. 1981. Los Crocodylia de Sur América. I. Los Crocodylia de Colombia. Carrera 7a , Bogotá, Colombia.

Pérez, T.A. 2000. Crecimiento del Caiman crocodilus crocodilus en cautiverio. Interciencia 25: 442-446.

Pérez, T.A. \& A. Velasco. 2002. Evaluación del crecimiento de Crocodylus intermedius con una dieta experimental. Bol. Centro Invest. Biol. 36: 125-135.

Pinheiro, M.S., S.A. Santos \& R. A. Silva. 1992. Efeito da temperatura da agua sobre o crescimento inicial de Caiman crocodilus yacare. Rev. Brasil. Biol. 52: 161-168.

Pooley, T. 1991. Bases para la crianza de cocodrilos en zonas remotas, p. 81-109. In F. Wayne King (ed.). Crianza de Cocodrilos: Información de la Literatura Científica Grupo de Especialistas en Cocodrilos, I.U.C.N, The World Conservation Union, Gland, Suiza.

Ramírez-Perilla, J.A. 1991. Efecto de la cantidad y frecuencia alimentaria de una dieta experimental sobre la tasa de crecimiento de un ejemplar juvenil de Crocodylus intermedius, Crocodylia: Crocodylidae. Caldasia 16: 531-538.

Ramo, C., B. Busto \& A. Utrera. 1992. Breeding and rearing the Orinoco crocodile Crocodylus intermedius in Venezuela. Biol. Conserv. 60: $101-108$.

Ross, C.A. \& W.E. Magnuson. 1989. Living Crocodilians, p. 58-63. In C. A. Ross, (ed.). Crocodiles and Alligators. Facts On File, Nueva York, EEUU.

Staton, M.A., jr., I.L. Brisbin \& G. M. Pesti. 1986. Feed formulation for alligator: An overview and initial studies. Proc. $8^{\text {th }}$ Working Meeting, I.U.C.N, Crocodile Specialists Group, Quito, Ecuador. 